\title{
Unidentifiable object in the right atrium
}

\author{
Yusuke Asakura ${ }^{1}$, Yuka Nakamichi-Itoh ${ }^{2}$, and Kikuko Mori ${ }^{2}$ \\ ${ }^{1}$ Department of Anesthesiology, ${ }^{2}$ Physiological Laboratory, Nagoya Kyoritsu Hospital, Nagoya, Japan
}

After obtaining an Institutional Review Board approval of Nagoya Kyoritsu Hospital, Aichi, Japan (approved at the 46th IRB conference of the hospital) and written, informed consent from the patient, we present a case with an unidentified object in the right atrium. A 63-year-old woman undergoing regular hemodialysis ( 5 years of peritoneal dialysis and 7 years of hemodialysis) due to chronic kidney disease with a past medical history of pyogenic spondylodiscitis and gastric cancer presented to the pain clinic of the department of anesthesiology with complaints of lower backache and pain in her left leg. Her laboratory findings included white blood cell count of $8,900 / \mu \mathrm{l}$, blood urea nitrogen $8.96 \mathrm{mg} / \mathrm{dl}$, creatinine $4.05 \mathrm{mg} / \mathrm{dl}, 2.4 \mathrm{mg} / \mathrm{dl}$ of C-reactive protein and she was positive for procalcitonin. Her blood examination was otherwise unremarkable, and blood culture negative for bacteremia. Recurrence of pyogenic spondylodiscitis was suspected, and she underwent an emergency magnetic resonance imaging (MRI) evaluation. Simultaneously, echocardiography was performed to rule out the presence of infective endocarditis, which revealed a fluttering object inside her right atrium (Video 1). The fluttering membranous structure was different from a typical vegetation of infective endocarditis, and a retrospective review of her echocardiography revealed the same structure, suggesting that it was Chiari network. The diagnosis of recurrent of pyogenic spondylodiscitis was made based on her MRI findings [1].

Chiari network, a congenital remnant of the right valve of the sinus venosus, is frequently associated with patent foramen ovale (PFO), and its prevalence is reported to be approximately $2 \%-4 \%$ in the population $[2,3]$. In addition to the significance of this knowledge to routine trans-esophageal echocardiogram during anesthesia for cardiac surgery, an increased prevalence of right-to-left shunt in patients with PFO accompanied with Chiari network has been reported [2], resulting in an increased risk of arterial embolism. Thus, cases with Chiari network must be treated similarly to those with congenital heart disease with right-to-left shunt. The presence of a micro air-bubble in the fluid infusion should be carefully avoided.

Since our present case provides a typical echocardiogram of Chiari network, it is presented herein as a tutorial.

\section{References}

1. Asakura Y. Just think about pyogenic spondylodiscitis before performing the epidural steroid injection for low back pain. Korean J Anesthesiol 2018; 71: 161-2.

2. Schneider B, Hofmann T, Justen MH, Meinertz T. Chiari's network: normal anatomic variant or risk factor for arterial embolic events? J Am Coll Cardiol 1995; 26: 203-10.

3. Moral S, Ballesteros E, Huguet M, Panaro A, Palet J, Evangelista A. Differential diagnosis and clinical implications of remnants of the right valve of the sinus venosus. J Am Soc Echocardiogr 2016; 29: 183-94.

Corresponding author: Yusuke Asakura, M.D., M.Sc., Ph.D.

Department of Anesthesiology, Nagoya Kyoritsu Hospital, 1-172 Hokke, Nakagawa-ku, Nagoya, Aichi, Japan

Tel: 81-52-362-5151, Fax: 81-52-353-9105, Email: yasakura@kaikou.or.jp

ORCID: https://orcid.org/0000-0003-3966-1757

This article includes supplemental video clips.

Received: July 7, 2018. Accepted: July 18, 2018.

Korean J Anesthesiol 2018 December 71(6): 488

https://doi.org/10.4097/kja.d.18.00194

(c) This is an open-access article distributed under the terms of the Creative Commons Attribution Non-Commercial License (http://creativecommons.org/ licenses/by-nc/4.0/), which permits unrestricted non-commercial use, distribution, and reproduction in any medium, provided the original work is properly cited. 